\title{
Quality of life assessment in advanced cancer patients treated at home, an inpatient unit, and a day care center
}

This article was published in the following Dove Press journal:

OncoTargets and Therapy

8 May 2014

Number of times this article has been viewed

\author{
Wojciech Leppert ${ }^{1}$ \\ Mikolaj Majkowicz ${ }^{2}$ \\ Maria Forycka' \\ Eleonora Mess ${ }^{3}$ \\ Agata Zdun-Ryzewska ${ }^{2}$ \\ 'Department of Palliative \\ Medicine, Poznan University of \\ Medical Sciences, Poznan, Poland; \\ ${ }^{2}$ Department of Quality of Life \\ Research, Gdansk Medical University, \\ Gdansk, Poland; ${ }^{3}$ Palliative Care \\ Nursing Department, Wroclaw \\ Medical University, Wroclaw, Poland
}

Aim of the study: To assess quality of life (QoL) in cancer patients treated at home, at an in-patient palliative care unit (PCU), and at a day care center (DCC).

Patients and methods: QoL was assessed in advanced cancer patients at baseline and after 7 days of symptomatic treatment using the European Organization for Research and Treatment of Cancer Quality of Life Questionnaire-Core 15-Palliative Care (EORTC QLQ-C15-PAL), the Edmonton Symptom Assessment System (ESAS), and the Karnofsky Performance Status (KPS) scale.

Results: A total of 129 patients completed the study, with 51 patients treated at home, 51 patients treated at the PCU, and 27 patients at DCC. In the EORTC QLQ-C15-PAL, improvement in functional and symptom scales was observed except in physical functioning and fatigue levels; patients at DCC had a better physical functioning, global QoL, appetite, and fatigue levels. In the ESAS, improvement in all items was found except for drowsiness levels, which was stable in patients treated at DCC and deteriorated in home and PCU patients. Higher activity, better appetite and well-being, and less drowsiness were observed in patients treated at DCC. KPS was better in DCC patients compared to those treated at home and at the PCU; the latter group deteriorated. Conclusions: QoL improved in all patient groups, with better results in DCC patients and similar scores in those staying at home and at the PCU. Along with clinical assessment, baseline age, KPS, physical and emotional functioning may be considered when assigning patients to care at a DCC, PCU, or at home.

Keywords: oncology, patient care, performance status, advanced cancer, palliative care, quality of life

\section{Introduction}

Quality of life (QoL) is a multidimensional concept that takes strongly into account patients' subjective assessment of their own situation in a given period of time. ${ }^{1}$ QoL evaluation is very important in patients with chronic diseases such as cancer, as in many patients cure is not possible. ${ }^{2}$ However, the concept of palliative care allows to provide symptom relief and a holistic approach to cancer patients' needs with psychological, social, and spiritual support aimed at achieving the highest possible QoL for patients and their families. ${ }^{3}$ Thus, QoL evaluation is critically important for patients with advanced cancer, and several validated instruments have been developed for this purpose. ${ }^{4}$

Although QoL in patients with advanced cancer has been assessed in numerous studies, there are limited data on comparison of QoL of patients with advanced cancer treated at home and at an in-patient palliative care unit (PCU). Moro et al compared
Correspondence: Wojciech Leppert Department of Palliative Medicine, Poznan University of Medical Sciences, Osiedle Rusa 25A, 6I-245 Poznan, Poland

Tel/Fax +48 6I 8738303

Email wojciechleppert@wp.pl 
patients treated at home and at the PCU. Seven days after admission to PCU, only anxiety improved significantly, while pain, activity, nausea, depression, and drowsiness slightly decreased. In the home patient group, pain, nausea, and well-being improved significantly compared to baseline. However, these differences might be associated with the different frequencies of evaluation: twice daily at the PCU and once a week in home patients. ${ }^{5}$

Strömgren et al compared QoL in palliative care patients treated at home, at the PCU, and at an outpatient clinic using validated instruments for QoL assessment. The authors found similar QoL in patients treated at home and at the PCU, and superior QoL in those treated as outpatients. ${ }^{6}$ Modonesi et al demonstrated benefits of admission to the PCU, as all Edmonton Symptom Assessment System items improved when comparing baseline and Day 7 results of the symptomatic treatment. ${ }^{7}$ However, Elmqvist et al found that in the last 3 months of life, all functioning aspects deteriorated, mostly 1-2 months before death. Levels of fatigue, dyspnea, and appetite loss increased significantly, while the majority of patients suffered from severe pain in the course of the study. ${ }^{8}$

In several studies, benefits of day care center (DCC) services for advanced cancer patients were found. ${ }^{9,10}$ To our knowledge, no studies compared QoL in patients at DCC with those treated at the PCU and at home. Therefore, the aim of the study was to compare QoL in patients with advanced cancer treated in different settings (home, DCC, and PCU) and to assess factors that influenced patients' classification of home care, DCC, and the PCU.

\section{Patients and methods Patients}

A total of 160 consecutive advanced cancer patients referred to the Chair and Department of Palliative Medicine in Poznan (PCU, home, and DCC) were invited to participate in the questionnaire study between July and December 2010. To enter the study, patients had to have been diagnosed with advanced cancer, be over 18 , and be able to communicate effectively with the staff. Patients with primary and secondary tumors of the central nervous system and with cognitive impairment were excluded from the study.

Patients admitted to the PCU were those who could not be treated at home due to symptom burden or social problems; patients were followed up with every day by physicians and nurses, with other staff members available depending on patients' needs. Patients treated at home were unable to attend the outpatient clinic; nurses visited them at home at least twice a week, physicians visited at least twice a month, and other team members visited the patients whenever it was necessary. Patients treated at DCC were able to attend DCC twice a week; follow-up with a nurse was provided at each visit, with physician follow-up twice a month and follow-up with other staff members upon patient request.

Two QoL measurements were conducted for all instruments (baseline and during the Day 7 follow-up) consisting of symptom management plus psychosocial and spiritual support; measurement collection was similar for all three patient groups. All patients provided written informed consent to the study, which was performed in accordance with the Declaration of Helsinki. The study protocol was approved by the Regional Bioethics Committee of Poznan University of Medical Sciences.

\section{Instruments}

The following instruments were used: the European Organization for Research and Treatment of Cancer Quality of Life Questionnaire-Core 15-Palliative Care (EORTC QLQ-C15-PAL), ${ }^{11}$ the Edmonton Symptom Assessment System (ESAS) ${ }^{12}$ for QoL evaluation, and the Karnofsky Performance Status scale (KPS) for the performance status assessment. ${ }^{13}$ The EORTC recommends the EORTC QLQC15-PAL for QoL assessment in patients with advanced cancer, as it is a short, valid, and reliable tool for QoL evaluation in this patient population. It was developed from the EORTC QLQ-C30 core questionnaire, which was designed for measuring QoL in oncology patients. ${ }^{14}$ The EORTC QLQ-C15-PAL consists of two functional scales: physical functioning (questions 1-3), emotional functioning (questions 13 and 14), and one global QoL item (question 15). Symptom scales consist of a pain scale (questions 5 and 12), fatigue scale (questions 7 and 11), and single items of dyspnea (4), insomnia (6), lack of appetite (8), nausea (9), and constipation (10). For questions 1-14, patients respond to a four-point Likert scale: 1) not at all, 2) a little, 3) quite a bit, and (4) very much. For question 15 (global QoL), patients respond to a seven-point numerical scale: 1) very poor overall QoL to 7) excellent overall QoL. Responses are related to the past week. EORTC QLQ-C15PAL psychometric properties were established in patients with advanced cancer. ${ }^{15-17}$ The EORTC QLQ-C15-PAL was adapted to the Polish clinical setting. ${ }^{18}$

The ESAS is a simple, valid, and reliable instrument designed for QoL evaluation in advanced cancer patients. The original version of the tool comprises ten items for symptom assessment (pain, activity, nausea, anorexia, 
well-being, dyspnea, depressive mood, anxiety, drowsiness, and an additional item for any symptom indicated by patients). ${ }^{12}$ The original version of the instrument has been recently modified. ${ }^{19}$ Two additional items were added to the original version of the ESAS for frequent symptoms assessment in advanced cancer patients: vomiting and constipation. ${ }^{20}$ All items were assessed on an eleven-point scale: 0 ) no symptom to 10 ) the most severe intensity of the symptom. Several studies demonstrated appropriate psychometric properties and the usefulness of the ESAS for the purpose of monitoring the QoL and quality of palliative care provided for patients with advanced cancer in different clinical settings. ${ }^{5-7,21}$ The Polish version of the ESAS was validated. ${ }^{22}$ The KPS is designed for the assessment of patients' performance status on the $0-100$ scale: 0 ) death to 100$)$ fully active. ${ }^{13}$

\section{Statistical analysis}

The scoring of the unchanged scales (pain) and single items (dyspnea, insomnia, appetite loss, and constipation) of the EORTC QLQ-C15-PAL was performed according to the EORTC QLQ-C30 Scoring Manual. ${ }^{23}$ The global QoL item of the EORTC QLQ-C15-PAL was scored as a single item following the procedures of the EORTC QLQ-C30 Scoring Manual. The EORTC QLQ-C15-PAL abbreviated scales (physical functioning, emotional functioning, nausea and vomiting, and fatigue) were estimated using the Addendum to the EORTC QLQ-C30 Scoring Manual: Scoring of the EORTC QLQ-C15-PAL. ${ }^{24}$

The quantitative data of all items of all the instruments used (the EORTC QLQ-C15-PAL, the ESAS, and the KPS) in all three settings (home care, PCU, and DCC) were expressed as arithmetic means and standard deviations. The scores of all patients at the first and second QoL evaluation of the EORTC QLQ-C15-PAL scales and single items were compared using the Student's $t$ test. The scores of the EORTC QLQ-C15-PAL, the ESAS, and the KPS in patients treated at home, in the PCU, and at DCC were compared using analysis of variance with repeated measures. In the case of significant main effects (patient group, measurement, interaction), a post hoc test was used to find differences between patient groups. A multivariate discriminant function analysis was conducted to establish factors that influenced patients' classification to home care, the PCU, and DCC. Data were statistically analyzed with Statistica version 9.1 (StatSoft, Inc., Tulsa, OK, USA). In all statistical analyses, a $P$-value of less than 0.05 was considered significant.

\section{Results}

Of 160 patients, 129 patients completed two QoL measurements, with ten patients refusing to participate. From 21 patients who dropped out, the condition of ten patients deteriorated significantly and they were unable to complete the instruments (six at the PCU and four at home), eight patients died (three at the PCU and five at home) and three patients at DCC were lost to follow-up (Figure 1). From 129 patients who completed the study, there were 51 patients treated at home and at the PCU each and 27 patients treated at DCC.

With respect to age, there were no differences between patients treated at DCC and the PCU and between DCC and home patients. However, a tendency was observed between the age of home and PCU patients $[F=3.065 ; P=0.0502]$. No difference was found between the age of all men and all women $[t(127)=0.218 ; P=0.877]$. Similarly, there was no difference in the age of men $(F=1.799 ; P=0.173)$ and women $(F=2.242$; $P=0.116)$ in all three patient groups. The distribution of primary tumor location in all three patient groups was similar ( chi-square $=18.759$; degrees of freedom $(\mathrm{df})=14 ; P=0.174$ ).

Referring to sex distribution, there was no difference between patients treated at home and at the PCU $[\mathrm{F}(1)=0.168$; $P=0.682]$. However, differences were found with respect to sex distribution between patients treated at DCC and

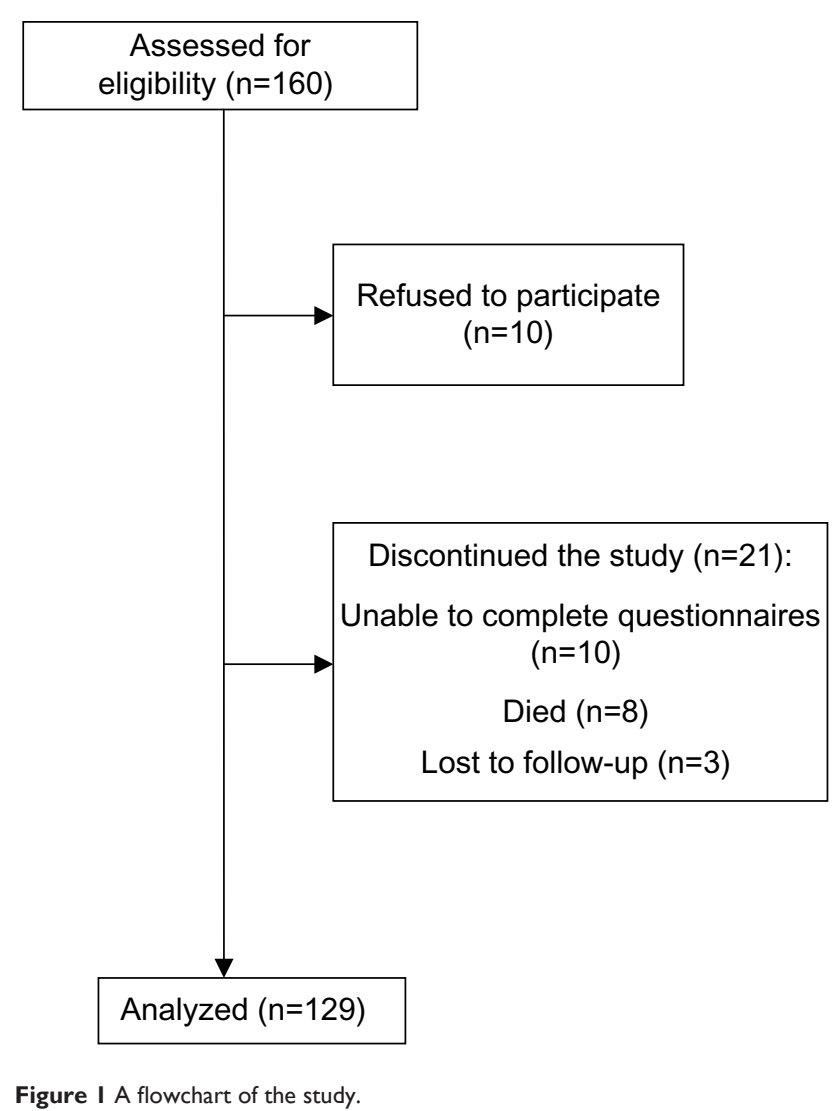


those treated at home and at the PCU [Pearson chi-square (1) $=11.539 ; P=0.002]$. Patients at DCC had better activity scores (KPS) compared to those treated at home and at the PCU (Table 1).

\section{EORTC QLQ-CI5-PAL}

The descriptive statistics and a comparison of the scores of the EORTC QLQ-C15-PAL scales and single items at the first and at the second QoL assessment for all patients are shown in Table 2. In all of the EORTC QLQ-C15-PAL scales and single items, significant improvement was observed $(P<0.001)$ except for physical functioning and fatigue. In the EORTC QLQ-C15-PAL functional scales, a patient group effect was found in physical functioning and global QoL $(P<0.0001)$. The measurement effect was noted in emotional functioning and global QoL $(P<0.0001)$ as both improved in all patient groups. No interaction effect was observed (analysis of variance) (Table 3 ). Physical functioning was stable in all patient groups; it was better in DCC compared to home and PCU $(P<0.0001)$ patients, with no difference between home and PCU (post hoc). Emotional functioning and global QoL improved in all patient groups; in the latter, better results were observed in DCC compared to home and PCU $(P<0.0001)$ patients, with no difference between home and PCU.
In the EORTC QLQ-C15-PAL symptom scales and single items, a patient group effect in fatigue $(P=0.0002)$ and loss of appetite $(P=0.012)$ was observed. The measurement effect in pain scale and in all single items (dyspnea, insomnia, loss of appetite, nausea, and constipation) was found $(P<0.0001)$, except for the fatigue scale; these symptoms improved in all patient groups. No interaction effect was observed (Table 4). Fatigue was stable in all patient groups, but it was less intense in DCC compared to home $(P<0.0001)$ and PCU patients $(P<0.0001 ; P=0.002$ the first and the second QoL evaluation, respectively), with no difference between home and PCU (post hoc). Appetite was better in DCC compared to PCU $(P<0.0001)$ and home patients in the first $(P=0.007)$ but not in the second QoL assessment, with no difference between home and PCU patients.

\section{ESAS and Karnofsky}

In all of the ESAS items, significant improvement was observed at the second compared to the first QoL assessment for all patients ( $P=0.001$ for drowsiness; $P<0.0001$ for all other items) (Table 5). ESAS results varied depending on the patient group in the case of activity, appetite, well-being, and drowsiness. In all ESAS items, a measurement effect was observed; all symptoms improved $(P<0.0001)$ except for drowsiness, which deteriorated $(P=0.011)$ in home and PCU patients and remained

Table I Demographic and clinical data of 129 patients

\begin{tabular}{|c|c|c|c|c|}
\hline Characteristics & $\begin{array}{l}\text { Palliative } \\
\text { care unit }\end{array}$ & Home care & $\begin{array}{l}\text { Day care } \\
\text { center }\end{array}$ & $P$-value \\
\hline \multicolumn{5}{|l|}{ Age (years) } \\
\hline All patients & $64.25 \pm 12.18$ & $70.18 \pm \mid 2.51$ & $67.4 I \pm I I .00$ & $0.502 *$ \\
\hline $67.30 \pm 12.32$ (range, 33-94) & (range, 44-86) & (range, 33-94) & (range, 44-89) & \\
\hline Men & $64.00 \pm 12.96$ & $68.97 \pm 12.32$ & $71.43 \pm 8.06$ & 0.173 \\
\hline $67.10 \pm 12.52$ (range, 33-89) & (range, 44-86) & (range, 33-88) & (range, 65-89) & \\
\hline Women & $64.65 \pm 11.20$ & $72.39 \pm 12.91$ & $66.00 \pm 11.71$ & 0.116 \\
\hline $67.50 \pm 12.18$ (range, 44-94) & (range, 45-85) & (range, 5I-94) & (range, 44-86) & $0.877^{\dagger}$ \\
\hline \multicolumn{5}{|l|}{ Sex } \\
\hline Men n=7I (55.0\%) & 31 (60.78\%) & 33 (64.7I\%) & $7(25.93 \%)$ & $0.002 * *$ \\
\hline Women $n=58$ (45.0\%) & $20(39.22 \%)$ & 18 (35.29\%) & $20(74.07 \%)$ & \\
\hline Karnofsky I & $47.45 \pm 9.77$ & $48.82 \pm 9.09$ & $68.15 \pm 14.95$ & $<0.00 I^{* *}$ \\
\hline Karnofsky 2 & $44.90 \pm 9.25$ & $47.06 \pm 10.45$ & $70.74 \pm 14.92$ & $<0.00 I^{* *}$ \\
\hline Primary tumor location & & & & 0.397 \\
\hline Lung n=26 (20.15\%) & 12 (23.53\%) & II (2I.57\%) & $3(11.11 \%)$ & \\
\hline Colon $n=19(14.73 \%)$ & $8(15.69 \%)$ & $8(15.69 \%)$ & $3(11.11 \%)$ & \\
\hline Kidney $n=12$ (9.30\%) & $5(9.80 \%)$ & $4(7.84 \%)$ & $3(|1| 1 \%)$. & \\
\hline Prostate $n=12(9.30 \%)$ & $6(11.76 \%)$ & $5(9.80 \%)$ & I (3.70\%) & \\
\hline Breast $n=9(6.98 \%)$ & $3(5.88 \%)$ & $\mathrm{I}(1.96 \%)$ & $5(18.52 \%)$ & \\
\hline Head and neck $n=8$ (6.20\%) & $4(7.84 \%)$ & $\mathrm{I}(1.96 \%)$ & $3(1 \mathrm{l} .1 \mathrm{l} \%)$ & \\
\hline Ovary $n=7(5.43 \%)$ & I (I.96\%) & $5(9.80 \%)$ & I (3.70\%) & \\
\hline Pancreas $n=7$ (5.43) & $2(3.92 \%)$ & $3(5.88 \%)$ & $2(7.41 \%)$ & \\
\hline Other $n=29(22.48 \%)$ & II (2I.87\%) & $13(25.49 \%)$ & $6(22.22 \%)$ & \\
\hline Total n= $229(100 \%)$ & $51(100 \%)$ & $51(100 \%)$ & $27(100 \%)$ & \\
\hline
\end{tabular}

Notes: *Home vs palliative care unit; ’all men vs all women; ${ }^{* *}$ day care center vs home and palliative care unit. 
Table 2 A descriptive statistics (means and standard deviations) and a comparison of the results of the EORTC QLQ-CI5-PAL for all patients

\begin{tabular}{|c|c|c|c|}
\hline $\begin{array}{l}\text { Item/scale } \\
\text { (item number) }\end{array}$ & Baseline & Day 7 & $P$-value* \\
\hline yysical functioning $(\mathrm{I}-3)$ & $24.19 \pm 22.14$ & $22.69 \pm 21.75$ & $P=0.107$ \\
\hline & & & \\
\hline in $(5,12)$ & $76.48 \pm 23.16$ & $27.65 \pm 13.26$ & $P<0.00$ I \\
\hline somnia (6) & $47.03 \pm 19.37$ & 41 & \\
\hline tigue $(7,1 \mathrm{I})$ & $60.30 \pm 20.04$ & $58.83 \pm 24.4 \mid$ & $P=0.405$ \\
\hline sss of appetite & $53.23 \pm 24.12$ & $34.1 I \pm 24.81$ & $P<0.001$ \\
\hline ausea and vomiting (9) & $23.19 \pm 24.67$ & $3.65 \pm 6.93$ & $P<0.001$ \\
\hline onstipation (10) & $53.49 \pm 30.74$ & $34.11 \pm 26.18$ & $P<0.001$ \\
\hline $\begin{array}{l}\text { Emotional } \\
\text { functioning }(13,14)\end{array}$ & $54.54 \pm 16.80$ & $68.56 \pm 14.28$ & $P<0.001$ \\
\hline Global quality of life (I5) & $37.47 \pm 10.42$ & $55.17 \pm 11.18$ & $P<0.00$ I \\
\hline \multicolumn{4}{|c|}{$\begin{array}{l}\text { Notes: I-3, I3-I5: Functional scales and single items of the EORTC QLQ-CI5- } \\
\text { PAL. Higher scores indicate better functioning and higher QoL. 4-12: Symptom } \\
\text { scales and single items of the EORTC QLQ-CI5-PAL. Higher scores indicate more } \\
\text { severe symptoms and lower QoL. *Student's t-test. } \\
\text { Abbreviations: EORTC QLQ-CI5-PAL, European Organization for Research and } \\
\text { Treatment of Cancer Quality of Life Questionnaire-Core I5-Palliative Care; QoL, } \\
\text { quality of life. }\end{array}$} \\
\hline
\end{tabular}

Table 3 A descriptive statistics (means and standard deviations) and a comparison of the EORTC QLQ-CI5-PAL functional scales scores

\begin{tabular}{llll}
\hline Functional & Baseline & Day 7 & P-value* \\
scales of the & & \\
EORTC & & \\
QLQ-CI5-PAL & & \\
\hline
\end{tabular}

\begin{tabular}{|c|c|c|c|}
\hline \multicolumn{4}{|c|}{ Physical functioning (I-3) } \\
\hline Palliative care unit & $|4.9| \pm|8.4|$ & $13.34 \pm 16.28$ & \\
\hline Home care & $|9.88 \pm| 6.4 \mid$ & $17.91 \pm 16.99$ & \\
\hline Day care center & $49.87 \pm 18.79$ & $49.37 \pm 17.57$ & \\
\hline Patient group & & & $<0.0001$ \\
\hline Measurement & & & 0.170 \\
\hline Interaction & & & 0.842 \\
\hline \multicolumn{4}{|c|}{ Emotional functioning $(13,14)$} \\
\hline Palliative care unit & $51.50 \pm 20.87$ & $67.83 \pm|6.4|$ & \\
\hline Home care & $56.73 \pm 12.25$ & $68.00 \pm 12.83$ & \\
\hline Day care center & $56.20 \pm 15.25$ & $71.01 \pm 12.73$ & \\
\hline Patient group & & & 0.385 \\
\hline Measurement & & & $<0.0001$ \\
\hline Interaction & & & 0.312 \\
\hline \multicolumn{4}{|c|}{ Global quality of life (15) } \\
\hline Palliative care unit & $35.62 \pm 10.55$ & $51.63 \pm 11.18$ & \\
\hline Home care & $35.62 \pm 8.18$ & $53.27 \pm 8.18$ & \\
\hline Day care center & $44.44 \pm 11.32$ & $65.43 \pm 10.26$ & \\
\hline Patient group & & & $<0.000$ I \\
\hline Measurement & & & $<0.000$ I \\
\hline Interaction & & & 0.206 \\
\hline
\end{tabular}

Notes: *P-value (analysis of variance with repeated measures); least significant differences test post hoc indicated on the differences between the day care center, palliative care unit, and home care. Higher scores indicate better functioning and better QoL.

Abbreviations: EORTC QLQ-C15-PAL, European Organization for Research and Treatment of Cancer Quality of Life Questionnaire-Core 15-Palliative Care; QoL, quality of life.
Table 4 A descriptive statistics (means and standard deviations) and a comparison of the EORTC QLQ-CI5-PAL symptom scales scores

\begin{tabular}{llll}
\hline $\begin{array}{l}\text { Symptom scales } \\
\text { of the EORTC } \\
\text { QLQ-CI5-PAL }\end{array}$ & Baseline & Day 7 & P-value* \\
\hline $\begin{array}{llll}\text { Fatigue (7, II) } \\
\text { Palliative care unit }\end{array}$ & $64.06 \pm 33.30$ & $60.78 \pm 33.30$ & \\
Home care & $63.64 \pm 15.58$ & $64.28 \pm 22.38$ & \\
$\begin{array}{l}\text { Day care center } \\
\text { Patient group }\end{array}$ & $46.90 \pm 20.77$ & $44.84 \pm 21.70$ & \\
Measurement & & & 0.0002 \\
Interaction & & & 0.399 \\
Nausea and vomiting (9) & & 0.609 \\
$\begin{array}{l}\text { Palliative care unit } \\
\text { Home care }\end{array}$ & $23.55 \pm 26.49$ & $4.58 \pm 7.53$ & \\
$\begin{array}{l}\text { Day care center } \\
\text { Patient group }\end{array}$ & $22.57 \pm 20.76$ & $3.60 \pm 6.94$ & \\
Measurement & & $1.86 \pm 5.35$ & \\
Interaction & & & 0.920 \\
Pain $(5,73.74$ & & $<0.000$ I \\
\end{tabular}

Pain (5, 12)

Palliative care unit $\quad 79.74 \pm 21.94 \quad 28.76 \pm 13.78$

Home care $\quad 75.49 \pm 22.45 \quad 27.78 \pm 12.32$

Day care center $\quad 72.22 \pm 26.55 \quad 25.3 \mathrm{I} \pm 14.15$

Patient group

Measurement

Interaction

Dyspnea (4)

Palliative care unit

Home care

0.373

$<0.000$ I

0.547

Day care center

Patient group

Measurement

Interaction

Insomnia (6)

Palliative care unit

Home care

Day care center

Patient group

Measurement

Interaction

Loss of appetite (8)

Palliative care unit

Home care

$28.10 \pm 30.82$

$13.73 \pm 17.86$

$29.4 I \pm 25.5|\quad| 5.69 \pm 16.80$

$24.69 \pm 19.81 \quad 17.28 \pm 16.97$

0.907

$<0.0001$

0.292

Day care center

Patient group

Measurement

Interaction

Constipation (10)

Palliative care unit

Home care

$55.56+27.22$

$37.25 \pm 31.02$

Day care center

(5.56 27.22

$35.29 \pm 23.49$

Patient group

$25.93 \pm 19.25$

0.304

$<0.000$ I

0.343

Measurement

0.115

$<0.000$ I

Interaction

0.853

Notes: ${ }^{*} P$-value (analysis of variance with repeated measures); least significant differences test post hoc indicated on the differences between the day care center and palliative care unit and home care. Higher scores indicate more intense symptoms and worse QoL. Abbreviations: EORTC QLQ-CI5-PAL, European Organization for Research and Treatment of Cancer Quality of Life Questionnaire-Core 15-Palliative Care; QoL, quality of life. 
Table 5 A descriptive statistics (means and standard deviations) and a comparison of the results of ESAS

\begin{tabular}{|c|c|c|c|}
\hline ESAS items & Baseline & Day 7 & $P$-value* \\
\hline \multicolumn{4}{|l|}{ Pain (I) } \\
\hline All patients & $6.28 \pm 1.76$ & $1.76 \pm 1.28$ & $<0.0001$ \\
\hline Palliative care unit & $6.49 \pm 1.80$ & $1.78 \pm 1.35$ & \\
\hline Home care & $6.12 \pm 1.63$ & $1.82 \pm 1.22$ & \\
\hline Day care center & $6.18 \pm 1.92$ & $1.59 \pm 1.28$ & \\
\hline Patient group & & & 0.679 \\
\hline Measurement & & & $<0.0001$ \\
\hline Interaction & & & 0.463 \\
\hline \multicolumn{4}{|l|}{ Activity (2) } \\
\hline All patients & $6.67 \pm 1.34$ & $4.23 \pm 1.76$ & $<0.0001$ \\
\hline Palliative care unit & $6.89 \pm 1.25$ & $4.88 \pm 1.79$ & \\
\hline Home care & $6.88 \pm 1.20$ & $4.47 \pm 1.36$ & \\
\hline Day care center & $5.86 \pm 1.50$ & $2.56 \pm 1.28$ & \\
\hline Patient group & & & $<0.0001$ \\
\hline Measurement & & & $<0.0001$ \\
\hline Interaction & & & 0.015 \\
\hline \multicolumn{4}{|l|}{ Nausea (3) } \\
\hline All patients & $2.89 \pm 2.31$ & $0.52 \pm 0.81$ & $<0.0001$ \\
\hline Palliative care unit & $2.78 \pm 2.35$ & $0.54 \pm 0.90$ & \\
\hline Home care & $3.09 \pm 2.09$ & $0.57 \pm 0.69$ & \\
\hline Day care center & $2.69 \pm 2.66$ & $0.39 \pm 0.86$ & \\
\hline Patient group & & & 0.677 \\
\hline Measurement & & & $<0.0001$ \\
\hline Interaction & & & 0.745 \\
\hline \multicolumn{4}{|l|}{ Vomiting (4) } \\
\hline All patients & $1.28 \pm 2.17$ & $0.32 \pm 0.52$ & 0.0001 \\
\hline Palliative care unit & $1.23 \pm 2.39$ & $0.3 I \pm 0.54$ & \\
\hline Home care & $1.34 \pm 1.89$ & $0.34 \pm 0.4 \mathrm{I}$ & \\
\hline Day care center & $1.26 \pm 2.29$ & $0.29 \pm 0.67$ & \\
\hline Patient group & & & 0.956 \\
\hline Measurement & & & $<0.0001$ \\
\hline Interaction & & & 0.977 \\
\hline \multicolumn{4}{|l|}{ Constipation (5) } \\
\hline All patients & $5.27 \pm 2.63$ & $3.4 I \pm 2.18$ & $<0.0001$ \\
\hline Palliative care unit & $5.60 \pm 2.92$ & $3.54 \pm 2.71$ & \\
\hline Home care & $5.26 \pm 2.52$ & $3.56 \pm 1.85$ & \\
\hline Day care center & $4.68 \pm 2.19$ & $2.89 \pm 1.55$ & \\
\hline Patient group & & & 0.306 \\
\hline Measurement & & & $<0.0001$ \\
\hline Interaction & & & 0.663 \\
\hline \multicolumn{4}{|l|}{ Appetite (6) } \\
\hline All patients: & $5.95 \pm 1.80$ & $3.86 \pm 1.86$ & $<0.0001$ \\
\hline Palliative care unit & $5.89 \pm 1.80$ & $4.14 \pm 2.15$ & \\
\hline Home care & $6.32 \pm 1.48$ & $3.99 \pm 1.45$ & \\
\hline Day care center & $5.35 \pm 2.20$ & $3.07 \pm 1.87$ & \\
\hline Patient group & & & 0.027 \\
\hline Measurement & & & $<0.0001$ \\
\hline Interaction & & & 0.278 \\
\hline \multicolumn{4}{|l|}{ Well-being (7) } \\
\hline All patients & $6.84 \pm|.3|$ & $3.91 \pm 1.23$ & $<0.0001$ \\
\hline Palliative care unit & $6.94 \pm 1.26$ & $4.21 \pm 1.43$ & \\
\hline Home care & $7.16 \pm 1.12$ & $4.13 \pm 0.79$ & \\
\hline Day care center & $6.03 \pm 1.43$ & $2.94 \pm 1.02$ & \\
\hline Patient group & & & $<0.0001$ \\
\hline Measurement & & & $<0.0001$ \\
\hline Interaction & & & 0.539 \\
\hline
\end{tabular}

(Continued)
Table 5 (Continued)

\begin{tabular}{|c|c|c|c|}
\hline ESAS items & Baseline & Day 7 & P-value* \\
\hline \multicolumn{4}{|l|}{ Dyspnea (8) } \\
\hline All patients & $2.35 \pm 2.21$ & $1.11 \pm 1.25$ & $<0.0001$ \\
\hline Palliative care unit & $2.4 I \pm 2.40$ & $1.03 \pm 1.14$ & \\
\hline Home care & $2.63 \pm 2.29$ & $\mathrm{I} .37 \pm \mathrm{I} .47$ & \\
\hline Day care center & $1.70 \pm 1.47$ & $0.78 \pm 0.87$ & \\
\hline Patient group & & & 0.153 \\
\hline Measurement & & & $<0.0001$ \\
\hline Interaction & & & 0.390 \\
\hline \multicolumn{4}{|l|}{ Depression (9) } \\
\hline All patients & $4.82 \pm 2.01$ & $2.79 \pm 1.52$ & $<0.0001$ \\
\hline Palliative care unit & $4.74 \pm 1.96$ & $2.8 I \pm I .64$ & \\
\hline Home care & $4.95 \pm 2.04$ & $2.92 \pm 1.37$ & \\
\hline Day care center & $4.76 \pm 2.09$ & $2.47 \pm 1.67$ & \\
\hline Patient group & & & 0.684 \\
\hline Measurement & & & $<0.0001$ \\
\hline Interaction & & & 0.670 \\
\hline \multicolumn{4}{|l|}{ Anxiety (10) } \\
\hline All patients & $4.30 \pm 1.93$ & $2.53 \pm 1.56$ & $<0.0001$ \\
\hline Palliative care unit & $4.32 \pm 2.00$ & $2.40 \pm 1.49$ & \\
\hline Home care & $4.21 \pm 2.03$ & $2.59 \pm 1.62$ & \\
\hline Day care center & $4.43 \pm 1.66$ & $2.66 \pm 1.60$ & \\
\hline Patient group & & & 0.882 \\
\hline Measurement & & & $<0.0001$ \\
\hline Interaction & & & 0.604 \\
\hline \multicolumn{4}{|l|}{ Drowsiness (I I) } \\
\hline All patients & $2.29 \pm 1.10$ & $2.78 \pm 1.52$ & 0.001 \\
\hline Palliative care unit & $2.46 \pm 1.02$ & $2.91 \pm 1.67$ & \\
\hline Home care & $2.28 \pm 1.17$ & $3.04 \pm 1.44$ & \\
\hline Day care center & $2.00 \pm 1.08$ & $1.98 \pm 1.08$ & \\
\hline Patient group & & & 0.018 \\
\hline Measurement & & & 0.011 \\
\hline Interaction & & & 0.113 \\
\hline
\end{tabular}

Notes: *P-value (analysis of variance with repeated measures); least significant differences test post hoc indicated on the differences between the day care center, palliative care unit, and home care. Higher scores indicate more intense symptoms, less activity, poorer well-being, and worse QoL.

Abbreviations: ESAS, Edmonton Symptom Assessment System; QoL, quality of life.

stable in DCC patients. Interaction effect was not observed in any ESAS items except for activity $(P=0.015)$ (Table 5). Appetite improved in all patient groups, with better scores in DCC compared to home $(P=0.025 ; P=0.033$ the first and the second QoL assessment, respectively) and $\mathrm{PCU}$ patients, but in the latter only in the second evaluation $(P=0.013)$ and with no difference between home and PCU patients (post hoc).

Well-being improved in all patient groups; it was better in DCC patients compared to those treated at home $(P<0.0001)$ and at the PCU $(P=0.002 ; P<0.0001)$, with no difference between home and PCU patients. Drowsiness intensified in home and PCU patients and remained stable in DCC patients; it was less intense in DCC patients compared to home $(P=0.0001)$ and PCU $(P=0.003)$ patients in the second evaluation, with no difference between patients staying at home and at the PCU. 
Table 6 Descriptive statistics (means and standard deviations) and a comparison of the results of the Karnofsky scale

\begin{tabular}{llll}
\hline Karnofsky & Baseline & Day 7 & P-value* \\
\hline All patients & $52.33 \pm 13.49$ & $51.16 \pm 14.98$ & 0.104 \\
Palliative care unit & $47.45 \pm 9.77$ & $44.90 \pm 9.25$ & \\
Home care & $48.82 \pm 9.09$ & $47.06 \pm 10.45$ & \\
Day care center & $68.15 \pm 14.95$ & $70.74 \pm 14.92$ & \\
Patient group & & & $<0.0001$ \\
Measurement & & & 0.431 \\
Interaction & & & 0.021 \\
\hline
\end{tabular}

Notes: *P-value (analysis of variance with repeated measures); the least significant differences test post hoc indicated the differences between the day care center, palliative care unit, and home care. Higher scores indicate better activity.

Activity assessed by the ESAS improved in all groups $(P<0.0001)$; patients at DCC had higher activity compared to other groups at both measurements $(P=0.002 ; P<0.0001$; for both in the first and the second measurements, respectively), with no difference between home and PCU patients (post hoc). In the staff assessment (KPS), patients at DCC displayed higher level of activity compared to home and PCU patients $(P<0.0001)$, with no difference between PCU and home patients (post hoc). Patient activity remained stable in DCC and in home patients, but deteriorated in those staying at the PCU $(P<0.0001)$. However, KPS did not change significantly in the whole group between the first and the second QoL assessment (Table 6).

A multivariate discriminant function analysis was conducted with predictors such as age, KPS scores, and QoL dimensions: physical functioning, emotional functioning, global QoL, fatigue, nausea and vomiting, pain, dyspnea, sleep, appetite and constipation. The following variables had statistically significant discriminant function: age $(P=0.042)$, KPS $(P=0.007)$, physical functioning $(P=0.046)$ and emotional functioning $(P=0.041)$. The prediction of a correct classification of all patients to any of the groups (treated at home, the PCU, or DCC) equals $64.06 \%$. The best prediction was for patients treated at DCC (69.23\%), slightly worse for those treated at home $(66.66 \%)$, and the lowest for patients treated at the PCU (58.82\%).

\section{Discussion}

In this study, QoL of advanced cancer patients treated at home, at the PCU, and at a DCC was assessed using the following validated instruments: the EORTC QLQ-C15-PAL, the ESAS, and the KPS. The results showed similar QoL in patients treated at home and at the PCU, and superior QoL in those treated at DCC. Patients treated at DCC had better physical functioning and global QoL, less fatigue and better appetite (EORTC QLQ-C15-PAL), higher activity, better appetite and well-being, and less drowsiness (ESAS) compared to home and PCU patients. However, PCU and home patients had similar QoL, with lower functioning level and higher symptom burden. After 7 days of treatment, symptoms improved, with the exception of physical functioning and fatigue (EORTC QLQ-C15-PAL), drowsiness (ESAS), and activity (KPS).

In the EORTC QLQ-C15-PAL functional scales, emotional functioning and global QoL improved in all patient groups. Emotional functioning level was similar in all patient groups. Global QoL was higher in DCC compared to home and PCU patients, with no difference between home and PCU patients. The improvement in emotional functioning was probably due to the psychological support offered to all patients and an effective symptomatic treatment. ${ }^{25}$ These factors probably also improved baseline global QoL scores in the second assessment. The physical functioning level was higher in DCC compared to home and PCU patients, with no difference between home and PCU patients. The fact that physical functioning did not improve during the study period in any of the patient groups might be explained by the advanced stage of cancer and its progression. ${ }^{8}$

Nearly all EORTC QLQ-C15-PAL symptom scales (pain) and single items (dyspnea, insomnia, lack of appetite, nausea and constipation) improved, with the exception of the fatigue scale. Differences between patient groups were observed with respect to fatigue and appetite, which were less intense in DCC patients compared to those staying at home and at the PCU, with similar results between the latter groups. A lack of improvement in fatigue may be due to the ineffectiveness of medical intervention in advanced cancer patients. ${ }^{26}$ Opposite to fatigue, appetite improvement might be associated with a more effective treatment during the study period. ${ }^{27}$

ESAS scores improved consistently with symptom relief observed in the EORTC QLQ-C15-PAL, with the exception of fatigue, but fatigue was not measured by the ESAS. However, drowsiness was the only ESAS item that intensified in home and PCU patients and remained stable in DCC patients. Less intense drowsiness in DCC patients compared to home and PCU patients was probably associated with the more severe condition of those treated at home and at the PCU. ${ }^{5}$ It may be supported by the fact that fatigue (EORTC QLQ-C15PAL) was less intense and KPS was better in DCC patients compared to home and PCU patients. Appetite (consistently with EORTC QLQ-C15-PAL) and well-being (assessed by the ESAS only) both improved in all patient groups, with better scores in DCC compared to home and PCU patients. This is probably associated with the aforementioned effective 
treatment of appetite loss and effective symptom management with psychosocial support, respectively.

Higher activity was consistently observed in both assessments in DCC patients in KPS and in ESAS compared to home and PCU patients. Although KPS decreased in PCU patients, the mean deterioration of 2.55 on a $0-100$ scale may be clinically irrelevant. ${ }^{28}$ It should be also noted that home patients also deteriorated by 1.76 , but this was statistically insignificant and the results were similar to PCU patients. It may also be noted that KPS scores did not change significantly for the whole group. In contrast to KPS scores, ESAS results have shown a significant and clinically relevant improvement in all three patient groups. ${ }^{29}$ This discrepancy between patients' (ESAS) and staff (KPS) activity assessment might be associated with patients' overestimation of their condition and an experience of significant relief in most of the physical symptoms, anxiety, depression, emotional functioning, well-being, and global QoL. It may also reflect a greater symptom burden in PCU patients, as this group comprised those who cannot be successfully treated at DCC or at home.

The differences observed in our study between patient groups indicate a lower symptom burden, better well-being, and higher activity in DCC patients compared to home and PCU patients. Our results are comparable to Strömgren et al, who found similar QoL in patients treated at home and at the PCU but superior QoL and better KPS scores in outpatients. ${ }^{6}$ Heedman and Strang found significant correlation between well-being and symptom distress ESAS scores in advanced cancer patients treated at home. ${ }^{21}$ Similar to our results, Modonesi et al demonstrated benefits of admission to PCU, as all ESAS items improved comparing baseline and Day 7 of therapy. ${ }^{7}$ However, according to Elmqvist et al, QoL of advanced cancer patients decreases in the last 3 months of life. ${ }^{8}$

Our study results indicate that patients treated at DCC have a better QoL compared to home and PCU patients. There is a gap in the literature regarding direct comparisons between these three palliative care settings. Our results suggest that apart from common palliative care provision at home and at the PCU, DCC programs should be strongly considered. This is further supported by the fact that early introduction of palliative and supportive care may be more effective and provide higher QoL in advanced cancer patients than a standard palliative care. ${ }^{30}$

A multivariate discriminant function analysis was conducted with predictors such as baseline age, KPS, and QoL scores. Significant discriminant functions were found for age, KPS, and physical and emotional functioning, with the highest correctly classified patients at a DCC (70\%) and lower for those treated at home (67\%) and at the PCU (59\%). Apart from individualized and meticulous clinical evaluation and patients' preferences, these variables may be taken into account when classifying patients to the most appropriate place of care in advanced cancer.

Several limitations of the study should be addressed, such as uncontrolled design, lack of a control group, patients' recruitment from one academic institution, and a modest patient sample that comprised diverse diagnoses of solid tumors. QoL was assessed within 7 days, without longer follow-up. Patients with cognitive impairment were excluded from the study, as were patients with brain tumors. Some patients dropped out due to deterioration or death, and some were lost to follow-up. Several factors might have influenced the obtained results, such as differences between patient groups with respect to age (a trend of older home patients vs PCU patients) and sex distribution (more women in DCC vs at home and PCU). Patients at a DCC had better baseline KPS scores compared to those treated at home and at the PCU.

\section{Conclusion}

QoL improved in all three patient groups comparing baseline and follow-up assessment on Day 7. QoL was superior in patients treated at DCC compared to those treated at home and at the PCU, with similar level in the latter groups. Apart from clinical evaluation and patient preferences, baseline age, KPS, physical functioning and emotional functioning may be considered when classifying advanced cancer patients to a DCC, home care or the PCU.

\section{Disclosure}

The authors declare no conflicts of interest in this work.

\section{References}

1. Schipper H. Quality of life: principles of the clinical paradigm. J Psychosocial Oncol. 1990;8(2-3):171-185.

2. De Walden-Galuszko K. Quality of life - general considerations. In: De Walden-Galuszko K, Majkowicz M, editors. Quality of Life in Cancer. Gdansk: Wydawnictwo Uniwersytetu Gdanskiego; 1994:13-40. Polish.

3. WHO definition of palliative care [webpage on the Internet]. World Health Organization, Geneva 2002. Available at: http://www.who.int/ cancer/palliative/definition/en/. Accessed February 26, 2014.

4. Kaasa S, Loge JH. Quality of life in palliative care: principles and practice. Palliat Med. 2003;17(1):11-20.

5. Moro C, Brunelli C, Miccinesi G, et al. Edmonton symptom assessment scale: Italian validation in two palliative care settings. Support Care Cancer. 2006;14(1):30-37.

6. Strömgren AS, Goldschmidt D, Groenvold M, et al. Self-assessment in cancer patients referred to palliative care: a study of feasibility and symptom epidemiology. Cancer. 2002;94(2):512-520. 
7. Modonesi C, Scarpi E, Maltoni M, et al. Impact of palliative care unit admission on symptom control evaluated by the edmonton symptom assessment system. J Pain Symptom Manage. 2005;30(4):367-373.

8. Elmqvist MA, Jordhøy MS, Bjordal K, Kaasa S, Jannert M. Healthrelated quality of life during the last three months of life in patients with advanced cancer. Support Care Cancer. 2009;17(2):191-198.

9. Low J, Perry R, Wilkinson S. A qualitative evaluation of the impact of palliative care day services: the experiences of patients, informal carers, day unit managers and volunteer staff. Palliat Med. 2005;19(1):65-70.

10. Stevens E, Martin CR, White CA. The outcomes of palliative care day services: a systematic review. Palliat Med. 2011;25(2):153-169.

11. Groenvold M, Petersen MA, Aaronson NK, et al; for the EORTC Quality of Life Group. The development of the EORTC QLQ-C15-PAL: A shortened questionnaire for cancer patients in palliative care. Eur $J$ Cancer. 2006;42(1):55-64.

12. Bruera E, Kuehn N, Miller MJ, Selmser P, Macmillan K. The Edmonton Symptom Assessment System (ESAS): a simple method for the assessment of palliative care patients. J Palliat Care. 1991;7(2):6-9.

13. Karnofsky DA, Burchenal JH. The clinical evaluation of chemotherapeutic agents in cancer. In: MacLeod CM, editor. Evaluation of Chemotherapeutic Agents, New York: Columbia University Press; 1949:191-205.

14. Aaronson NK, Ahmedzai S, Bergman B, et al. The European Organization for Research and Treatment of Cancer QLQ-C30: a quality-of-life instrument for use in international clinical trials in oncology. J Natl Cancer Inst. 1993;85(5):365-376.

15. Suárez-del-Real Y, Allende-Pérez S, Alférez-Mancera A, et al. Validation of the Mexican-Spanish version of the EORTC QLQC15-PAL questionnaire for the evaluation of health-related quality of life in patients on palliative care. Psychooncology. 2011;20(8): 889-896.

16. Shin DW, Choi JE, Miyashita M, et al. Cross-cultural application of the Korean version of the European Organization for Research and Treatment of Cancer Quality of Life Questionnaire-Core 15-Palliative Care. J Pain Symptom Manage. 2011;41(2):478-484.

17. Miyazaki K, Suzukamo Y, Shimozuma K, Nakayama T. Verification of the psychometric properties of the Japanese version of the European Organization for Research and Treatment of Cancer Quality of Life Questionnaire Core 15 palliative (EORTCQLQ-C15-PAL). Qual Life Res. 2012;21(2):335-340.

18. Leppert W, Majkowicz M. Validation of the Polish version of the European Organization for Research and Treatment of Cancer Quality of Life Questionnaire - Core 15 - Palliative Care in patients with advanced cancer. Palliat Med. 2013;27(5):470-477.
19. Watanabe SM, Nekolaichuk C, Beaumont C, Johnson L, Myers J, Strasser F. A multicenter study comparing two numerical versions of the Edmonton Symptom Assessment System in palliative care patients. J Pain Symptom Manage. 2011;41(2):456-468.

20. Leppert W, Majkowicz M. The impact of tramadol and dihydrocodeine treatment on quality of life of patients with cancer pain. Int J Clin Pract. 2010;64(12):1681-1687.

21. Heedman PA, Strang P. Symptom assessment in advanced palliative home care for cancer patients using the ESAS: clinical aspects. Anticancer Res. 2001;21(6A):4077-4082.

22. Majkowicz M, Czuszynska Z, Leppert W, Maszkowska-Kopij K. Practical application of ESAS (Edmonton Symptom Assessment System) in palliative care. Nowotwory. 1998;48:847-857. Polish.

23. Fayers PM, Aaronson NK, Bjordal K, Groenvold M, Curran D, Bottomley A. The EORTC QLQ-C30 Scoring Manual. 3rd ed. Brussels, Belgium: European Organization for Research and Treatment of Cancer; 2001.

24. Groenvold M, Petersen MA; on behalf of the EORTC Quality of Life Group. Addendum to the EORTC QLQ-C30 Scoring Manual: Scoring of the EORTC QLQ-C15-PAL. Brussels, Belgium: EORTC Quality of Life Unit; 2006.

25. Rhondali W, Yennurajalingam S, Ferrer J, Chisholm G, Filbet M, Bruera E. Association between supportive care interventions and patient self-reported depression among advanced cancer outpatients. Support Care Cancer. 2013.

26. Radbruch L, Strasser F, Elsner F, et al; Research Steering Committee of the European Association for Palliative Care (EAPC). Fatigue in palliative care patients - an EAPC approach. Palliat Med. 2008;22(1): 13-32

27. Fearon K, Arends J, Baracos V. Understanding the mechanisms and treatment options in cancer cachexia. Nat Rev Clin Oncol. 2013;10(2):90-99.

28. Osoba D, Rodrigues G, Myles J, Zee B, Pater J. Interpreting the significance of changes in health-related quality-of-life scores. J Clin Oncol. 1998;16(1):139-144.

29. Selby D, Cascella A, Gardiner K, et al. A single set of numerical cutpoints to define moderate and severe symptoms for the Edmonton Symptom Assessment System. J Pain Symptom Manage. 2010;39(2):241-249.

30. Glare PA. Early implementation of palliative care can improve patient outcomes. J Natl Compr Canc Netw. 2013;11 Suppl 1:S3-S9.
OncoTargets and Therapy

\section{Publish your work in this journal}

OncoTargets and Therapy is an international, peer-reviewed, open access journal focusing on the pathological basis of all cancers, potential targets for therapy and treatment protocols employed to improve the management of cancer patients. The journal also focuses on the impact of management programs and new therapeutic agents and protocols on

\section{Dovepress}

patient perspectives such as quality of life, adherence and satisfaction. The manuscript management system is completely online and includes a very quick and fair peer-review system, which is all easy to use. Visit http://www.dovepress.com/testimonials.php to read real quotes from published authors. 